Public Abstract

Rachael Guerra

Ph.D.

Counseling Psychology

Influence of Racial Identity and Information Processing Strategies on Client Conceptualization

Advisor: Dr. Puncky Heppner

Graduation Term Summer 2005

\title{
Advisor's Signature
}

Several models exist that explain how counselors can more effectively work with racial/ethnic minority populations and how counselor characteristics (like racial identity) can impact the way counselors practice. Helms (1985) hypothesized that individuals operating in different statuses of racial identity will process racial information in ways characteristic of that status. Gushue and Carter (2000) found that the racial identity status of White participants was related to how they remembered stereotypical information about a hypothetical person. This study is an extension of Gushue and Carter's study with a sample of White counselors-in-training that investigated the relationship between participants' racial identity, their memory for racial stereotypes and their conceptualization of this client's presenting problems. Participants read a vignette about a Black hypothetical client and rated their conceptualization of his concerns. A week later, they completed a recognition task and racial identity attitude measures. Correlations indicated no relationship between racial identity attitudes scales and recognition of previously read stereotypical information, but did indicate that some racial identity attitudes were related to how the client was conceptualized. This finding reinforces the importance of multicultural counselor training. It is of great importance that counselors-in-training have opportunities to grow into the advanced stages of racial identity. 